To be submitted to The Astrophysical Journal

\title{
Equipotential Surfaces and Lagrangian points in Non-synchronous, Eccentric Binary and Planetary Systems
}

\author{
J. F. Sepinsky, B. Willems, V. Kalogera \\ j-sepinsky, b-willems, and vicky@northwestern.edu \\ Department of Physics and Astronomy, Northwestern University, 2145 Sheridan Road, \\ Evanston, IL 60208
}

\begin{abstract}
We investigate the existence and properties of equipotential surfaces and Lagrangian points in non-synchronous, eccentric binary star and planetary systems under the assumption of quasi-static equilibrium. We adopt a binary potential that accounts for non-synchronous rotation and eccentric orbits, and calculate the positions of the Lagrangian points as functions of the mass ratio, the degree of asynchronism, the orbital eccentricity, and the position of the stars or planets in their relative orbit. We find that the geometry of the equipotential surfaces may facilitate non-conservative mass transfer in non-synchronous, eccentric binary star and planetary systems, especially if the component stars or planets are rotating super-synchronously at the periastron of their relative orbit. We also calculate the volume-equivalent radius of the Roche lobe as a function of the four parameters mentioned above. Contrary to common practice, we find that replacing the radius of a circular orbit in the fitting formula of Eggleton (1983) with the instantaneous distance between the components of eccentric binary or planetary systems does not always lead to a good approximation to the volumeequivalent radius of the Roche-lobe. We therefore provide generalized analytic fitting formulae for the volume-equivalent Roche lobe radius appropriate for nonsynchronous, eccentric binary star and planetary systems. These formulae are accurate to better than $1 \%$ throughout the relevant 2 -dimensional parameter space that covers a dynamic range of 16 and 6 orders of magnitude in the two dimensions.
\end{abstract}

Subject headings: Celestial Mechanics, Stars: Binaries: Close, Stars: Planetary Systems 


\section{Introduction}

The Roche model has served for a long time as a fundamental tool in the study of the interactions and observational characteristics of the components of gravitational two-body systems. Among the most noteworthy applications are the modeling of the shapes of binary components, the study of mass transfer in interacting binaries, and the orbital stability of satellites in planetary systems. At the basis of these applications are the properties of the potential governing the gravitational and centrifugal forces operating in the system. In particular, the shape of equipotential surfaces and the existence of stationary points (where the net force exerted on a test particle vanishes) play a central role in our understanding of the evolution of binary star and planetary systems.

More often than not, applications of the Roche model are built on the assumption that the orbit of the system is circular and that the system components are rotating synchronously with the orbital motion. Under this assumption, the components can be treated as static with respect to a co-rotating frame of reference and their shapes are determined by equipotential surfaces of the system. However, the assumption of circular orbits and synchronous rotation cannot always be justified, neither on observational nor on theoretical grounds.

Observational support for non-circular orbits and non-synchronous rotation is very well established at present. Catalogs of eclipsing binaries as early as those by Shapley (1913) list binaries with non-negligible eccentricities ranging from 0.01 to 0.14 . The sample as well as the largest measured orbital eccentricity has since increased drastically (e.g. Struve 1950; Petrova \& Orlov 1999; Bildsten et al. 1997; Hegedüs et al. 2005; Pourbaix et al. 2004) and now also includes binaries in the Small and Large Magellanic Clouds (e.g. Bayne et al. 2002; Clausen et al. 2003; Bavne et al. 2004; Hilditch et al. 2005). Evidence for non-synchronous rotation of the components of close binary systems is equally abundant, and points to both sub- and supersynchronously rotating component stars (e.g. Struve 1950; Levato 1974; Habets \& Zwaan 1989; Meibom et al. 2006; van Hamme \& Wilson 1990).

Non-circular orbits are also observed in extrasolar planetary systems. Since the discovery of the first extrasolar planet orbiting a solar-type star, 51 Peg, (Mavor \& Queloz 1995; Marcy \& Butler 1995), the sample of known exoplanets has grown to more than 200, many of which orbit their host star with non-negligible orbital eccentricities. The most eccentric exoplanetary orbit known to date is that of HD 20782b which has an eccentricity of 0.9 (Jones et al. 2006). Constraints on exoplanetary rotation rates are yet to be inferred from observations.

Theoretically, circularization and synchronization processes are driven by tidal inter- 
actions between the components of binary or planetary systems 1 . Predicted circularization and synchronization time scales, however, depend strongly on the initial system parameters and theoretical uncertainties in the strength of tidal dissipation mechanisms (Mathieu et al. 2004; Meibom \& Mathieu 2005; Meibom et al. 2006). In addition, since synchronization tends to occur faster than circularization, components of binary and planetary systems tend to become synchronized with the orbital motion near periastron before the completion of circularization. The stellar and planetary rotation rates then deviate from synchronism at all other orbital phases.

Such deviations from synchronism in gravitational two-body systems induce time-dependent oscillations in the atmospheres of the component stars or planets (Zahn 1970, 1975). Therefore these objects can no longer be treated as static with respect to a rotating frame of reference, unless the time scale of the oscillatory motions is sufficiently long compared to the dynamical time scale of the star or planet. The validity of this approximation was first discussed by Limber (1963) for the case of non-synchronous circular binaries (see also Kruszewski 1963; Savonije 1978). He concluded that as long as the rotational angular velocities of the stars do not deviate considerably from synchronicity, the components may be approximated as static and their shapes may be determined by the instantaneous equipotential surfaces of the binary. Limber (1963) also found the approximation to be valid in the limiting case of stars rotating with angular velocities close to the break-up angular velocity due to the dominance of the centrifugal force over the gravitational forces.

Plaved (1958) was the first to abandon the assumption of synchronous rotation and study the effects of the asynchronicity on the shapes of the binary components and the position of the inner Lagrangian point $L_{1}$ (the stationary point of the Roche potential located in between the two stars on the line connecting their centers of mass) in binaries with circular orbits. He found that the position of the $L_{1}$ point tends to move closer to the mass center of a rapidly rotating binary component with increasing values of its rotational angular velocity. Correspondingly, the critical radius at which one component starts to transfer mass to its companion decreases with increasing rotational angular velocity. Later investigations extended the work of Plaved (1958) by more carefully considering the validity of the assumptions underlying the Roche model (Limber 1963; Kruszewski 1963) and considering the effect of spin-orbit misalignment in circular binaries (Avni \& Schiller 1982). Lubow (1979) also incorporated the effects of gas dynamics and heat transport in the surface layers of the binary components.

\footnotetext{
${ }^{1}$ For very close systems, orbital angular momentum losses due to gravitational wave emission may also contribute to the circularization process (Peters 1964).
} 
Avni (1976) further generalized the Roche model to account for eccentric binary orbits with the aim of modeling the light and radial-velocity variations observed in the eclipsing binary pulsar Vela X-1. His generalized binary potential was subsequently used by Wilson (1979) to discuss the computation of light and radial-velocity curves of eccentric binaries with non-synchronously rotating component stars. Regös et al. (2005), performed smoothed particle hydrodynamics calculations to validate the application of the Roche model to eccentric binaries with component stars rotating close to synchronism with the orbital angular velocity at periastron. Part of their work, however, is flawed due to an incorrect term in the expression for the gravitational potential (see $\S[$ for details).

Despite the vast observational support for the occurrence of eccentric orbits in binary and exoplanetary systems, a detailed account of the properties of the generalized Roche potential used to study these systems seems to be lacking from the literature. The geometry of the equipotential surfaces as well as the existence, location, and potential height of Lagrangian points are necessary, for instance, in determining when mass transfer is initiated in non-synchronous, eccentric binaries along with the determining how mass and angular momentum flow during these mass-transfer phases. Consequently the theoretical study of component interactions in non-synchronous binaries of arbitrary eccentricity requires the understanding of this geometry and properties of the binary potential. Such interactions (e.g., mass exchange, mass and angular momentum loss) very often occur as binaries evolve and their modeling enters almost all population synthesis calculations of binaries of current interest (X-ray binaries, gamma-ray burst progenitors, binary compact objects, etc.). They are also relevant to the evolution of planetary systems along with the possibility of mass transfer and loss from planets in orbit around their host stars. The pursuit of theoretical understanding of these interaction processes in the context of broad binary evolution studies is what motivates the study presented here.

The plan of the paper is as follows. In \$2, we introduce the Roche potential describing the motion of mass elements in non-synchronous, eccentric binary and planetary systems. In \$3, we discuss the time-dependence of the potential and investigate the conditions under which the potential varies sufficiently slowly to be considered quasi-static. Adopting these conditions, the existence and stability of stationary (Lagrangian) points of the potential are examined in $\$ 4$, In \$5, we calculate the volume-equivalent radius of the Roche Lobe as a function of the system parameters and provide a generalization of the Eggleton (1983) fitting formula appropriate for non-synchronous, eccentric binary star and planetary systems. In \$6, we examine the height of the potential along the line connecting the mass centers of the stellar or planetary binary components and discuss the implications for mass transfer and mass loss from the system. The final section, $\S 7$ is devoted to concluding remarks. 


\section{The Roche Potential for Non-Synchronous Eccentric Binaries}

We consider a binary system of stard 2 with masses $M_{1}$ and $M_{2}$ orbiting one another in a fixed Keplerian orbit with period $P_{\text {orb }}$, semi-major axis $a$, and eccentricity $e$. The distance $D$ between the mass centers of the two stars and the Keplerian orbital angular velocity $\omega_{K}$ of the stars are, at any time $t$, given by

$$
D(t)=\frac{a\left(1-e^{2}\right)}{1+e \cos \nu}
$$

and

$$
\omega_{K}(t)=\frac{2 \pi}{P_{\text {orb }}} \frac{(1+e \cos \nu)^{2}}{\left(1-e^{2}\right)^{3 / 2}}
$$

where $\nu$ is the true anomaly.

We assume the first star (hereafter star 1 ) rotates uniformly 3 about an axis perpendicular to the orbital plane with a fixed rotational angular velocity $\vec{\Omega}_{1}$ parallel to and directed in the same sense as the orbital angular velocity, $\vec{\omega}_{K}$. We furthermore assume star 1 is sufficiently centrally condensed to be approximated by a point mass surrounded by a uniformly rotating zero-density envelope. We assume this envelope is convectively stable so that no bulk motions of the mass elements of star 1 occur other than those due to the star's rotation and the tidal force exerted by the companion. The companion star (hereafter star 2) is treated as a point mass.

\footnotetext{
${ }^{2}$ For brevity, we will simply refer to the system components as stars, bearing in mind that the analysis is valid for planetary systems as well.

${ }^{3}$ The assumption of uniform rotation is made because it is the simplest possible, though it is not without merit. Spruit \& Phinney (1998) and Spruit (1999) argue that magnetic fields could establish uniform rotation. On the other hand, there are compelling cases of single and binary stars for which differential rotation has been claimed (See, e.g., Popper \& Plaved 1976; Domiciano de Souza et al. 2003).
} 


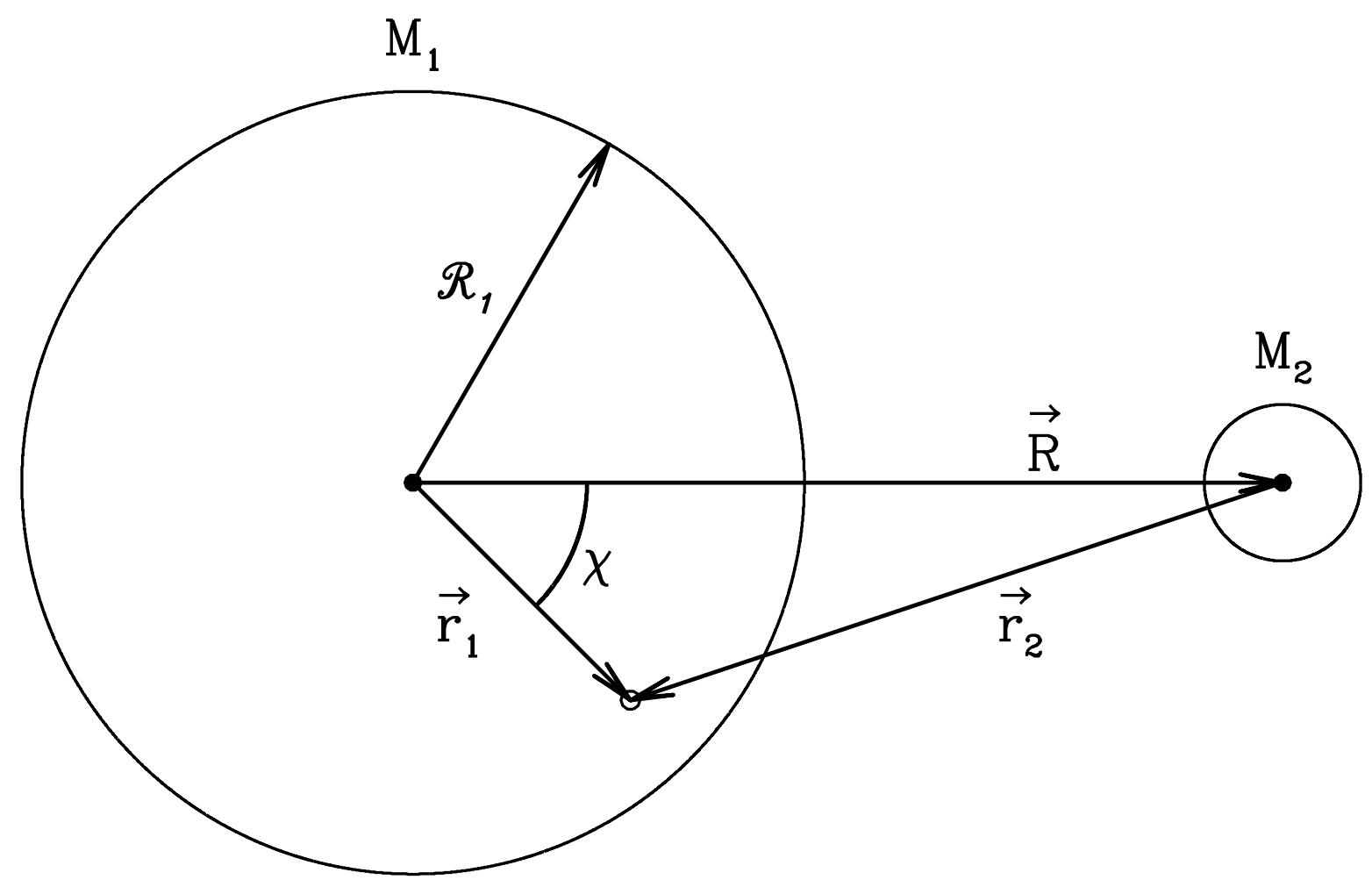

Fig. 1. - Schematic representation of the position vectors used in the derivation of the equation of motion of a mass element (open circle) in a component of a non-synchronous eccentric binary. 
The mass elements of star 1 are, at any given time, subjected to a sum of gravitational and rotational forces caused by the stars' mutual gravitational attraction, rotation, and orbital motion. We describe the motion of the mass elements of star 1 with respect to a Cartesian coordinate frame $O X Y Z$ with origin $O$ located at the center of mass of star 1 and $Z$-axis parallel to $\vec{\Omega}_{1}$. We furthermore let the frame rotate about the $Z$-axis with the rotational angular velocity of star 1 . The equation of motion of a mass element of star 1 with respect to $O X Y Z$ is then given by

$$
\ddot{\vec{r}}_{1}=-\frac{1}{\rho} \vec{\nabla} P-\vec{\nabla} V_{1}-2 \vec{\Omega}_{1} \times \dot{\vec{r}}_{1}
$$

where $\vec{r}_{1}$ is the position vector of the mass element with respect to the mass center of star 1 , $\rho$ and $P$ are the mass density and pressure of the mass element, and

$$
V_{1}=-G \frac{M_{1}}{\left|\vec{r}_{1}\right|}-G \frac{M_{2}}{\left|\vec{r}_{2}\right|}-\frac{1}{2}\left|\vec{\Omega}_{1}\right|^{2}\left(X^{2}+Y^{2}\right)+G \frac{M_{2} X}{D^{2}}
$$

is the potential giving rise to the gravitational and centrifugal forces acting on the mass element (for details, see Sepinsky et al. 2007, and references therein). Here, $G$ is the Newtonian constant of gravitation, $\vec{r}_{2}$ is the position vector of the considered mass element with respect to the mass center of star 2, and $X$ and $Y$ are the Cartesian coordinates of the mass element in the plane of the orbit.

We note that Eq. (4) is valid only when the $X$-axis of the reference frame coincides with the line connecting the mass centers of the binary components. Since the initial orientation of the $X$-and $Y$-axes in the orbital plane is arbitrary, the $X$-axis may be chosen to coincide with the line connecting the mass centers of the component stars at any given time without loss of generality. The definitions of the position vectors introduced in Eqs. (3) and (4) are shown schematically in Fig. 1.

The potential $V_{1}$ can be rewritten by expanding the gravitational attraction exerted by star 2 on the considered mass element of star 1 in terms of Legendre polynomials $P_{n}(x)$ as

$$
-G \frac{M_{2}}{\left|\overrightarrow{r_{2}}\right|}=-G \frac{M_{2}}{|\vec{R}|} \sum_{n=0}^{\infty}\left(\frac{\left|\vec{r}_{1}\right|}{|\vec{R}|}\right)^{n} P_{n}(\cos \chi)
$$

where $\vec{R}=\vec{r}_{1}-\vec{r}_{2}$ is the position vector of the mass center of star 2 with respect to the mass center of star 1 , and $\chi$ is the angle between $\vec{R}$ and $\vec{r}_{1}$. The first term in the expansion of $-G M_{2} /\left|\vec{r}_{2}\right|$ is independent of the position of the considered mass element and thus does not contribute to the force exerted on the element. The second term in the expansion of $-G M_{2} /\left|\vec{r}_{2}\right|$ is identical to the last term in Eq. (4). Equation (3) is therefore equivalent to

$$
\ddot{\vec{r}}_{1}=-\frac{1}{\rho} \vec{\nabla} P-\vec{\nabla} V_{1}^{*}-2 \vec{\Omega}_{1} \times \dot{\vec{r}}_{1},
$$


where

$$
V_{1}^{*}=-G \frac{M_{1}}{\left|\vec{r}_{1}\right|}-\frac{1}{2}\left|\vec{\Omega}_{1}\right|^{2}\left(X^{2}+Y^{2}\right)+W\left(\vec{r}_{1}, t\right) .
$$

In the latter equation, $W\left(\vec{r}_{1}, t\right)$ is the tide-generating potential defined as

$$
W\left(\vec{r}_{1}, t\right)=-G \frac{M_{2}}{|\vec{R}|} \sum_{n=2}^{\infty}\left(\frac{\left|\vec{r}_{1}\right|}{|\vec{R}|}\right)^{n} P_{n}(\cos \chi)
$$

(e.g., Zahn 1970; Polfliet \& Smevers 1990).

Denoting the radius of star 1 by $\mathcal{R}_{1}$ and restricting the tide-generating potential to its dominant $n=2$ terms, it follows from dimensional analysis that

$$
\frac{(1 / 2)\left|\vec{\Omega}_{1}\right|^{2}\left(X^{2}+Y^{2}\right)}{G M_{1} /\left|\vec{r}_{1}\right|} \sim\left(\frac{\left|\vec{\Omega}_{1}\right|}{\Omega_{c}}\right)^{2},
$$

and

$$
\frac{W}{G M_{1} /\left|\vec{r}_{1}\right|} \sim\left(\frac{\mathcal{R}_{1}}{D}\right)^{3} \frac{M_{2}}{M_{1}},
$$

where $\Omega_{c} \sim\left(G M_{1} / \mathcal{R}_{1}^{3}\right)^{1 / 2}$ is the break-up angular velocity of star 1 . The centrifugal distortion thus becomes increasingly important when $\left|\vec{\Omega}_{1}\right| \rightarrow \Omega_{c}$, while the tidal distortion becomes important when $\mathcal{R}_{1} \rightarrow D$ and $M_{2} \rightarrow M_{1}$.

\section{The quasi-static approximation}

For a binary with a circular orbit in which star 1 is rotating synchronously with the orbital motion, the potential $V_{1}$ is independent of time and the mass elements of star 1 are at rest with respect to the co-rotating frame of reference. Equation (3) therefore reduces to the condition for hydrostatic equilibrium

$$
\frac{1}{\rho} \vec{\nabla} P=-\vec{\nabla} V_{1},
$$

so that surfaces of constant pressure and density coincide with surfaces of constant $V_{1}$. The possible shapes of star 1 are thus determined by the equipotential surfaces of the binary.

For a binary with an eccentric orbit or non-synchronously rotating component stars, $V_{1}$ is a periodic function of time. At any given time, the mass elements of star 1 therefore undergo tidally induced oscillations on a time scale $\tau_{\text {tide }}$ determined by the difference between the instantaneous orbital angular velocity $\omega_{K}(t)$ and the rotational angular velocity $\left|\vec{\Omega}_{1}\right|$ :

$$
\tau_{\text {tide }}=\frac{2 \pi}{\left|\omega_{K}(t)-\right| \vec{\Omega}_{1}||} .
$$


From dimensional analysis, it follows that

$$
\begin{gathered}
\frac{\left|\ddot{\vec{r}}_{1}\right|}{|(\vec{\nabla} P) / \rho|} \sim \frac{\left|\ddot{\vec{r}}_{1}\right|}{\left|\vec{\nabla} V_{1}\right|} \sim \frac{\tau_{\text {dyn }}^{2}}{\tau_{\text {tide }}^{2}}, \\
\frac{\left|\vec{\Omega}_{1} \times \dot{\vec{r}}_{1}\right|}{|(\vec{\nabla} P) / \rho|} \sim \frac{\left|\vec{\Omega}_{1} \times \dot{\vec{r}}_{1}\right|}{\left|\vec{\nabla} V_{1}\right|} \sim \frac{\left|\vec{\Omega}_{1}\right|}{\Omega_{c}} \frac{\tau_{\text {dyn }}}{\tau_{\text {tide }}},
\end{gathered}
$$

where $\tau_{\text {dyn }}=\left(G M_{1} / \mathcal{R}_{1}^{3}\right)^{-1 / 2}$ is the dynamical time scale of star 1 . Hence, when $\tau_{\text {dyn }} \ll \tau_{\text {tide }}$, the $\ddot{\vec{r}}_{1}$ and $\vec{\Omega}_{1} \times \dot{\vec{r}}_{1}$ terms in Eq. (3) are negligible compared to the other terms, so that Eq. (3i) approximately reduces to Eq. (111). In this limiting case, the motions of the mass elements are so slow that, at each instant, star 1 can be considered to be quasi-static with respect to the rotating frame of reference $O X Y Z$. The instantaneous shape of the star can then be approximated by the instantaneous surfaces of constant $V_{1}$. Limber (1963) referred to this as the first approximation (see also Savonije 1978; Wilson 1979).

The condition that $\tau_{\text {dyn }} \ll \tau_{\text {tide }}$ can be expressed in terms of the orbital elements and properties of star 1 by writing $\tau_{\text {tide }}$ as

$$
\tau_{\text {tide }}=\frac{2 \pi}{\omega_{P}}\left|\left(\frac{1+e \cos \nu}{1+e}\right)^{2}-f\right|^{-1},
$$

where

$$
\omega_{P}=\frac{2 \pi}{P_{\mathrm{orb}}} \frac{(1+e)^{1 / 2}}{(1-e)^{3 / 2}}
$$

is the orbital angular velocity at periastron, and $f=\left|\vec{\Omega}_{1}\right| / \omega_{P}$ is the rotational angular velocity of star 1 in units of $\omega_{P}$. It follows that $\tau_{\text {dyn }} \ll \tau_{\text {tide }}$ when

$$
\frac{P_{\text {orb }}}{\tau_{\text {dyn }}} \gg \alpha(e, f, \nu)
$$

where $\alpha$ is a function of the orbital eccentricity $e$, the ratio of rotational to orbital angular velocity at periastron $f$, and the true anomaly $\nu$ :

$$
\alpha(e, f, \nu)=\frac{(1+e)^{1 / 2}}{(1-e)^{3 / 2}}\left|\left(\frac{1+e \cos \nu}{1+e}\right)^{2}-f\right| .
$$




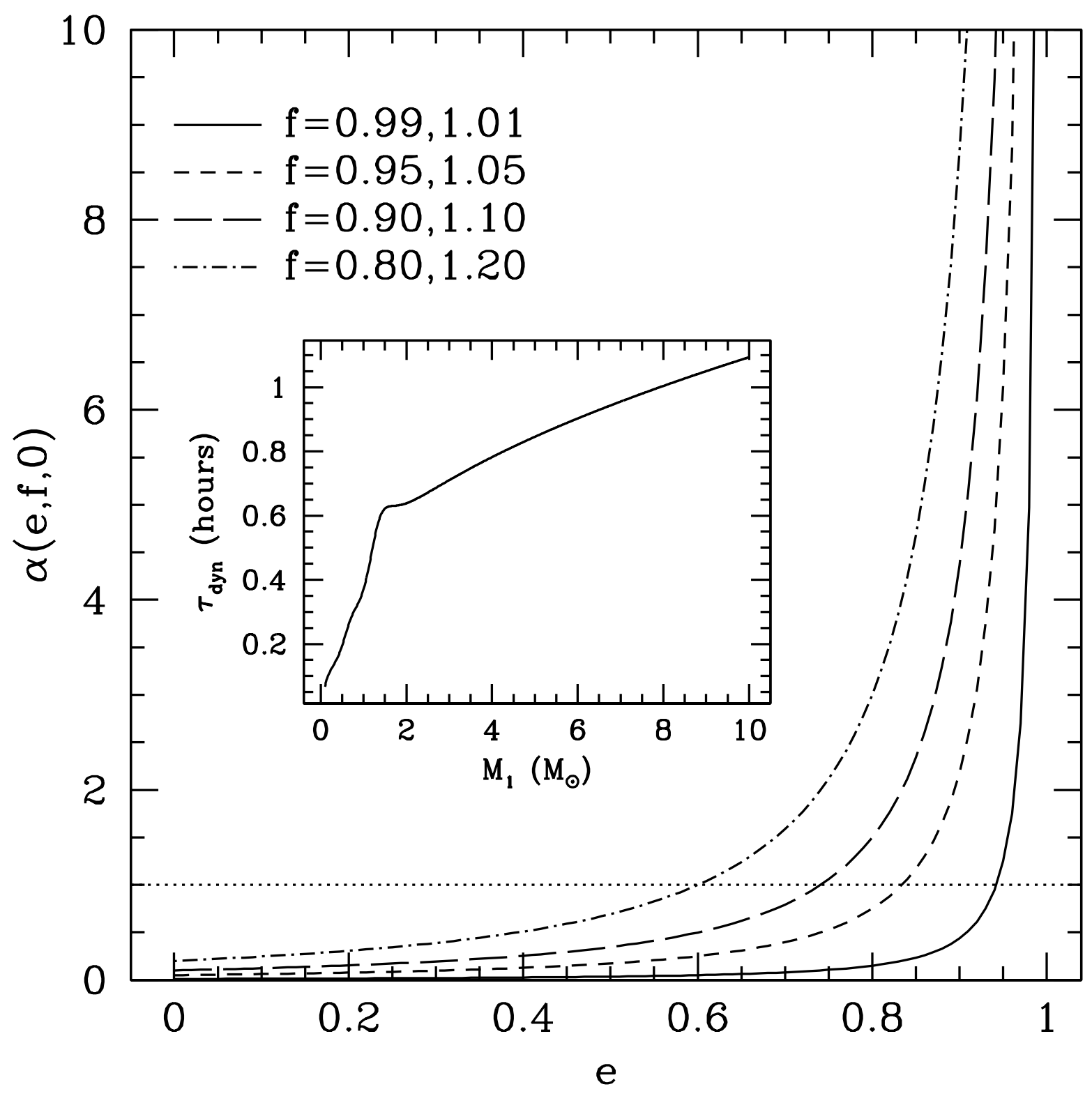

Fig. 2.- Variations of $\alpha(e, f, \nu)$ as a function of $e$, for $\nu=0$ and $0.8 \leq f \leq 1.2$. Due to the symmetry of $\alpha(e, f, 0)$ about $f=1$, the curves associated with $f=0.80,0.90,0.95,0.99$ are identical to those associated with $f=1.20,1.10,1.05,1.01$, respectively. For $\alpha(e, f, 0) \leq 1$ (below the dotted line), the quasi-static approximation is valid when $P_{\text {orb }} \gg \tau_{\text {dyn }}$. The inset shows the variation of $\tau_{\text {dyn }}$ as a function of stellar mass for stars on the ZAMS according to the mass-radius relation of Tout et al. (1996). 
When the companion is at the periastron of the relative orbit, $\alpha(e, f, \nu)$ reduces to

$$
\alpha(e, f, 0)=\frac{(1+e)^{1 / 2}}{(1-e)^{3 / 2}}|1-f| .
$$

Hence, when $f=1$ and the binary component stars are located at the periastron of their relative orbit, star 1 can be safely assumed to be quasi-static for all values of the orbital eccentricity $e<1$. However, once $f$ deviates from unity, the rapid increase of $\omega_{P}$ with increasing orbital eccentricity causes $\alpha(e, f, 0)$ to also increase rapidly with increasing orbital eccentricity. This is illustrated in more detail in the main panel of Fig. 2, For the considered range of $f$-values, the function $\alpha(e, f, 0)$ is smaller than 1 for $e \lesssim 0.6$. Hence, in this parameter range, the quasi-static approximation is valid at the periastron of the binary orbit if $P_{\text {orb }} \gg \tau_{\text {dyn }}$. As is illustrated by the inset of Fig. 2, this condition is easily satisfied for zero-age main sequence (ZAMS) stars of mass $M_{1} \lesssim 10 M_{\odot}$, provided the orbital period is longer than approximately $10 \mathrm{hr}$. For $M_{1} \lesssim 1 M_{\odot}$ this condition may be relaxed even further. Due to the nonlinear dependence of $\alpha(e, f, \nu)$ on $\nu$, the constraint on $P_{\text {orb }}$ may be more or less restrictive away from the periastron of the relative orbit.

\section{Lagrangian Points}

\subsection{Existence and Location}

In the case of a binary with a circular orbit and synchronously rotating component stars, there are five points at which the gravitational forces exactly balance the centrifugal forces. A mass element with zero velocity experiences no accelerations at these points and thus remains stationary with respect to the co-rotating frame of reference $O X Y Z$. Three of these so-called Lagrangian points lie on the line connecting the mass centers of the binary

components, and two lie on the tips of equilateral triangles whose bases coincide with the line connecting the mass centers of the component stars.

For binaries with eccentric orbits and/or non-synchronously rotating component stars, the tide-generating potential introduces a time-dependence in the potential $V_{1}$ and thus in the position of the stationary points (assuming these points still exist). To examine the existence and properties of these points, we adopt the quasi-static approximation, $\tau_{\text {dyn }} \ll \tau_{\text {tide }}$, and introduce the following dimensionless quantities:

$$
\begin{gathered}
V_{D}=\frac{V_{1}}{G M_{2} / D}, \quad q=\frac{M_{1}}{M_{2}}, \quad r_{D}=\frac{\left|\vec{r}_{1}\right|}{D}, \\
X_{D}=\frac{X}{D}, \quad Y_{D}=\frac{Y}{D}, \quad Z_{D}=\frac{Z}{D} .
\end{gathered}
$$


From Eq. (44), it follows that

$$
\begin{aligned}
V_{D} & =X_{D}-\frac{q}{r_{D}}-\frac{1}{\left(r_{D}^{2}-2 X_{D}+1\right)^{1 / 2}} \\
& -\frac{1}{2}\left(X_{D}^{2}+Y_{D}^{2}\right)(1+q) \mathcal{A}(f, e, \nu),
\end{aligned}
$$

where

$$
\mathcal{A}(f, e, \nu)=\frac{f^{2}(1+e)^{4}}{(1+e \cos \nu)^{3}} .
$$

This factor groups all dependencies of the potential on the degree of asynchronism, the orbital eccentricity, and the mean anomaly. In the particular case of a binary with a circular orbit and synchronously rotating component stars, $\mathcal{A}=1$.

The dependence of $\mathcal{A}(f, e, \nu)$ on $e$ and $\nu$ is illustrated in Fig. [3 for $f=1$. Since $\mathcal{A}(f, e, \nu)$ is linearly proportional to the square of the rotational angular velocity in units of the orbital angular velocity at periastron, the curves are easily rescaled for different values of $f$. For a given value of $\nu, \mathcal{A}(f, e, \nu)$ increases with increasing $e$, while for a given $e, \mathcal{A}(f, e, \nu)$ reaches a maximum at apastron and decreases towards periastron. $\mathcal{A}(f, e, \nu)$ is furthermore most sensitive to the value of the orbital eccentricity near $\nu=\pi$, where it increases from $\mathcal{A}(f, e, \nu)=1$ for $e=0$ to $\mathcal{A}(f, e, \nu) \approx 10^{4}$ for $e=0.9$. Moreover, at periastron, $\mathcal{A}(f, e, \nu) \leq 2$ for all $e<1$ and $f \leq 1$. 


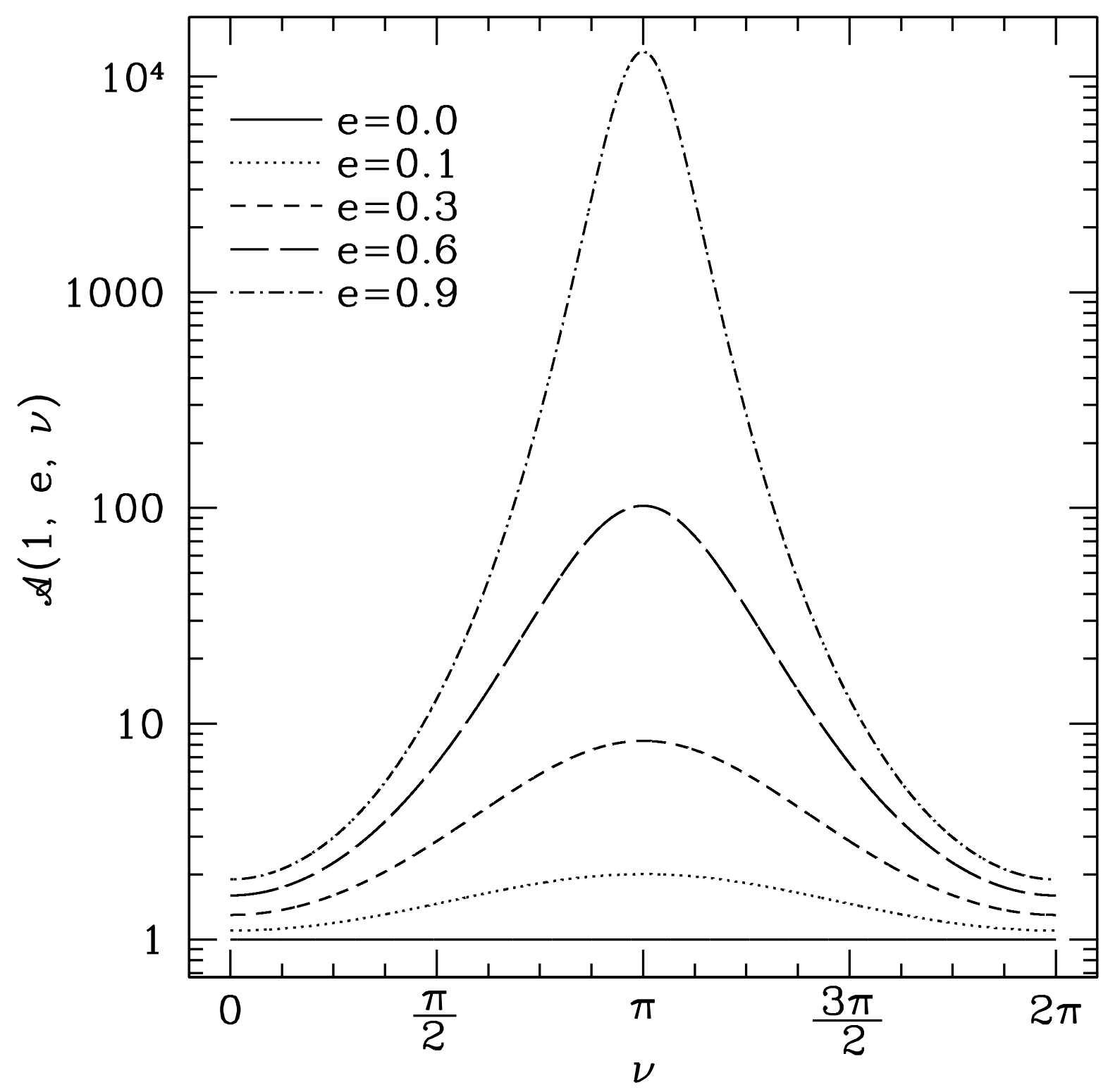

Fig. 3.- Variations of $\mathcal{A}(f, e, \nu)$ as a function $e$ and $\nu$, for $f=1$. Variations of $\mathcal{A}(f, e, \nu)$ for other values of $f$ are obtained by rescaling the plotted curves by a factor of $f^{2}$. 
The stationary points of the binary are solutions of the vector equation $\vec{\nabla} V_{D}=0$ with respective $X, Y$, and $Z$ components

$$
\begin{gathered}
X_{D}\left[\frac{q}{r_{D}^{3}}+\frac{1}{\left(r_{D}^{2}-2 X_{D}+1\right)^{3 / 2}}-(1+q) \mathcal{A}(f, e, \nu)\right] \\
-\frac{1}{\left(r_{D}^{2}-2 X_{D}+1\right)^{3 / 2}}+1=0, \\
Y_{D}\left[\frac{q}{r_{D}^{3}}+\frac{1}{\left(r_{D}^{2}-2 X_{D}+1\right)^{3 / 2}}\right. \\
\quad-(1+q) \mathcal{A}(f, e, \nu)]=0, \\
Z_{D}\left[\frac{q}{r_{D}^{3}}+\frac{1}{\left(r_{D}^{2}-2 X_{D}+1\right)^{3 / 2}}\right]=0,
\end{gathered}
$$

where we have used the fact that $r_{D}^{2}-2 X_{D}+1=\left(X_{D}-1\right)^{2}+Y_{D}^{2}+Z_{D}^{2}$ is a positive quantity. Since both terms inside the square brackets in Eq. (24) are positive, the equation can only be satisfied by setting $Z_{D}=0$. Thus, any and all stationary points necessarily lie in the plane of the orbit, as in the case for circular binaries with synchronously rotating component stars. We note in passing that Lagrangian points outside the orbital plane have been shown to exist when the spin axis of star 1 is inclined with respect to the orbital angular momentum axis of the binary (see, e.g., Avni \& Schiller 1982; Matese \& Whitmire 1983).

Letting $Z_{D}=0$, we solve Eqs. (22) and (23) for $X_{D}$ and $Y_{D}$ by distinguishing between two possible cases: $Y_{D}=0$ and $Y_{D} \neq 0$.

(i) $Y_{D}=0$. Setting $Y_{D}=Z_{D}=0$ reduces Eq. (22) to

$$
q \frac{X_{D}}{\left|X_{D}\right|^{3}}+\frac{X_{D}-1}{\left|X_{D}-1\right|^{3}}-X_{D}(1+q) \mathcal{A}(f, e, \nu)+1=0
$$

Leaving aside the trivial solution $X_{D}=0$, this equation has three real solutions which can only be determined numerically and which correspond to three co-linear stationary points on the line connecting the mass centers of the binary components. In accordance with the nomenclature for circular synchronized binaries, we will refer to these points as the Lagrangian points $L_{1}, L_{2}$, and $L_{3}$. The $L_{1}$ point is the stationary point located between the two component stars $\left(0<X_{D}<1\right)$, the $L_{2}$ point is the stationary point located opposite star 2 as seen from star $1\left(X_{D}>1\right)$, and the $L_{3}$ point is the stationary point located opposite star 1 as seen from star $2\left(X_{D}<0\right)$. 

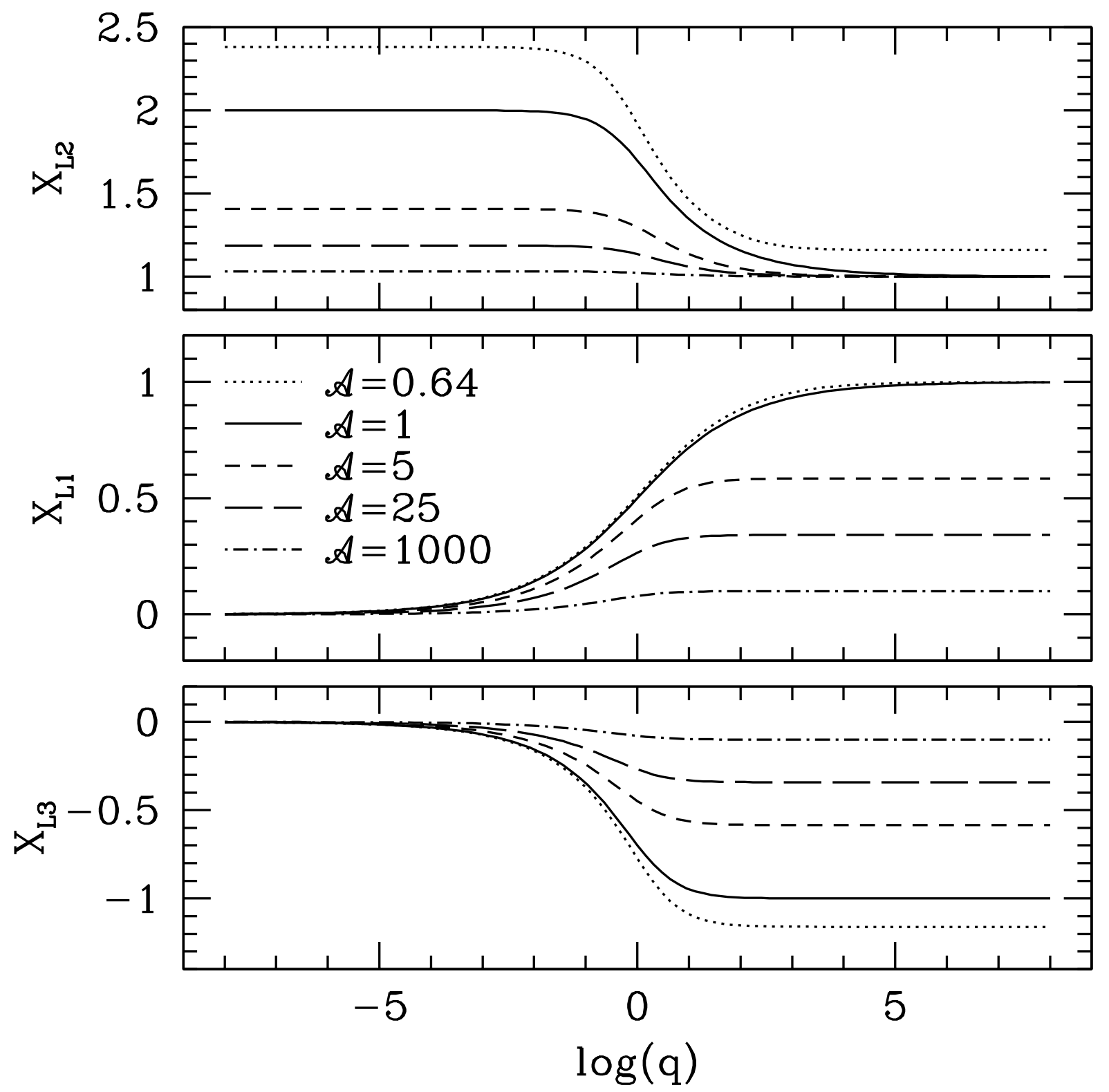

Fig. 4.- The position the co-linear Lagrange points $L_{1}, L_{2}$, and $L_{3}$ along the line connecting the mass centers of the binary components as a function of the logarithm of the mass ratio, $q$, for different values of $\mathcal{A}$. 
The location of each of the three co-linear Lagrangian points on the $X$-axis of the $O X Y Z$ frame is shown in Fig. 4 as a function of $q$ and $\mathcal{A}$. From the middle panel of Fig. 4 we can see that, for a given value of $\mathcal{A}, X_{L 1}$ increases with increasing values of $q$. For a given value of $q$, on the other hand, $X_{L 1}$ decreases with increasing values of $\mathcal{A}$. Additionally, $X_{L 1}$ asymptotes to a constant value for both large and small $q$. This behavior can be understood from Eq. (25), which, for small $q$, reduces to

$$
X_{L 1}\left[\mathcal{A}\left(X_{L 1}-1\right)^{2}-\left(X_{L 1}-1\right)+1\right] \approx 0 .
$$

The only real solution to this equation for which $0<X_{L 1}<1$ is $X_{L 1} \approx 0$. Thus, in the limit of small $q, X_{L 1} \rightarrow 0$. For large $q$, Eq. (25) yields

$$
X_{L 1} \approx \mathcal{A}^{-1 / 3} \text {. }
$$

This approximation breaks down for $\mathcal{A} \lesssim 1$, in which case $X_{L 1} \rightarrow 1$ for large values of $q$. This change in the behavior of $X_{L 1}$ for large values of $q$ is due to the fact that the gravitational force exerted by star 2 is no longer negligible as $X_{L 1} \rightarrow 1$.

For $L_{2}$, we can see from the top panel of Fig. 团 that, for a given value of $\mathcal{A}, X_{L 2}$ decreases with increasing values of $q$, and, for a given value of $q, X_{L 2}$ decreases with increasing values of $\mathcal{A}$. For small and large values of $q, X_{L 2}$ furthermore asymptotes to a constant value depending on the magnitude of $\mathcal{A}$. This may be understood from Eq. (25) which, for small $q$, reduces to

$$
X_{L 2}^{3}-\frac{2 \mathcal{A}+1}{\mathcal{A}} X_{L 2}^{2}+\frac{\mathcal{A}+2}{\mathcal{A}} X_{L 2}-\frac{2}{\mathcal{A}} \approx 0
$$

This equation admits of one real solution for $X_{L 2}$ which is a function of $\mathcal{A}$. The analytical expression for the solution is, however, rather intricate and does not contribute further to understanding the behavior of $X_{L 2}$ for small values of $q$ other than confirming the dependence of the asymptotic value of $X_{L 2}$ on $\mathcal{A}$. For large values of $q$, Eq. (25) yields

$$
X_{L 2} \approx \mathcal{A}^{-1 / 3} \text {. }
$$

This approximation breaks down for $\mathcal{A} \gtrsim 1$ for the same reason that Eq. (27) breaks down for $\mathcal{A} \lesssim 1$, i.e., the gravitational force exerted by star 2 is no longer negligible when $X_{L 2} \rightarrow 1$. Instead $X_{L 2} \approx 1$ for large values of $q$ and $\mathcal{A} \gtrsim 1$.

In the case of $L_{3}$, we can see from the bottom panel of Fig. 4 that, for a given value of $\mathcal{A}, X_{L 3}$ decreases with increasing values of $q$, while, for a given value of $q, X_{L 3}$ increases with increasing values of $\mathcal{A}$. In addition, $X_{L 3}$ asymptotes to zero for small values of $q$, and to a constant depending on $\mathcal{A}$ for large values of $q$. This may again be understood from Eq. (25) which, for small values of $q$, reduces to

$$
X_{L 3}\left[\mathcal{A} X_{L 3}^{2}-(2 \mathcal{A}+1) X_{L 3}+\mathcal{A}+2\right] \approx 0 .
$$


The only real solution to this equation with $X_{L 3}<0$ is $X_{L 3} \approx 0$. For large values of $q$, on the other hand, Eq. (25) yields

$$
X_{L 3} \approx-\mathcal{A}^{-1 / 3}
$$

for all values of $\mathcal{A}$.

(ii) $Y_{D} \neq 0$. For $Y_{D} \neq 0$ and $Z_{D}=0$, Eqs. (22) and (23) yield two non-linear, algebraic equations:

$$
\begin{gathered}
\frac{1}{\left(r_{D}^{2}-2 X_{D}+1\right)^{3 / 2}}-1=0, \\
\frac{q}{r_{D}^{3}}+\frac{1}{\left(r_{D}^{2}-2 X_{D}+1\right)^{3 / 2}}-(1+q) \mathcal{A}(f, e, \nu)=0 .
\end{gathered}
$$

These equations have two real, analytical solutions for $X_{D}$ and $Y_{D}$ given by

$$
\begin{aligned}
X_{D} & =\frac{1}{2}\left[\frac{q}{(1+q) \mathcal{A}(f, e, \nu)-1}\right]^{2 / 3} \\
Y_{D} & = \pm \sqrt{X_{D}\left(2-X_{D}\right)}
\end{aligned}
$$

The solutions correspond to two stationary points located on the tips of triangles whose bases coincide with the line connecting the mass centers of the binary component stars. In accordance with the nomenclature for circular synchronized binaries, we refer to these points as the triangular Lagrangian points $L_{4}$ and $L_{5}$. The $L_{4}$ point is the stationary point associated with $Y_{D}>0$, while the $L_{5}$ point is the stationary point associated with $Y_{D}<0$. Real solutions to Eqs. (34) and (35) furthermore only exist when

$$
q>\frac{1-\mathcal{A}}{\mathcal{A}-1 / 8}
$$

This condition is always satisfied when $\mathcal{A} \geq 1$. In the limiting case where $e=0$ and $f=1$, Eqs. (34) and (35) reduce to the usual formulae for the position of the $L_{4}$ and $L_{5}$ points in the case of a circular, synchronized binary, i.e. $X_{D}=1 / 2$ and $Y_{D}= \pm \sqrt{3} / 2$ (e.g. Murray \& Dermott 2000). 

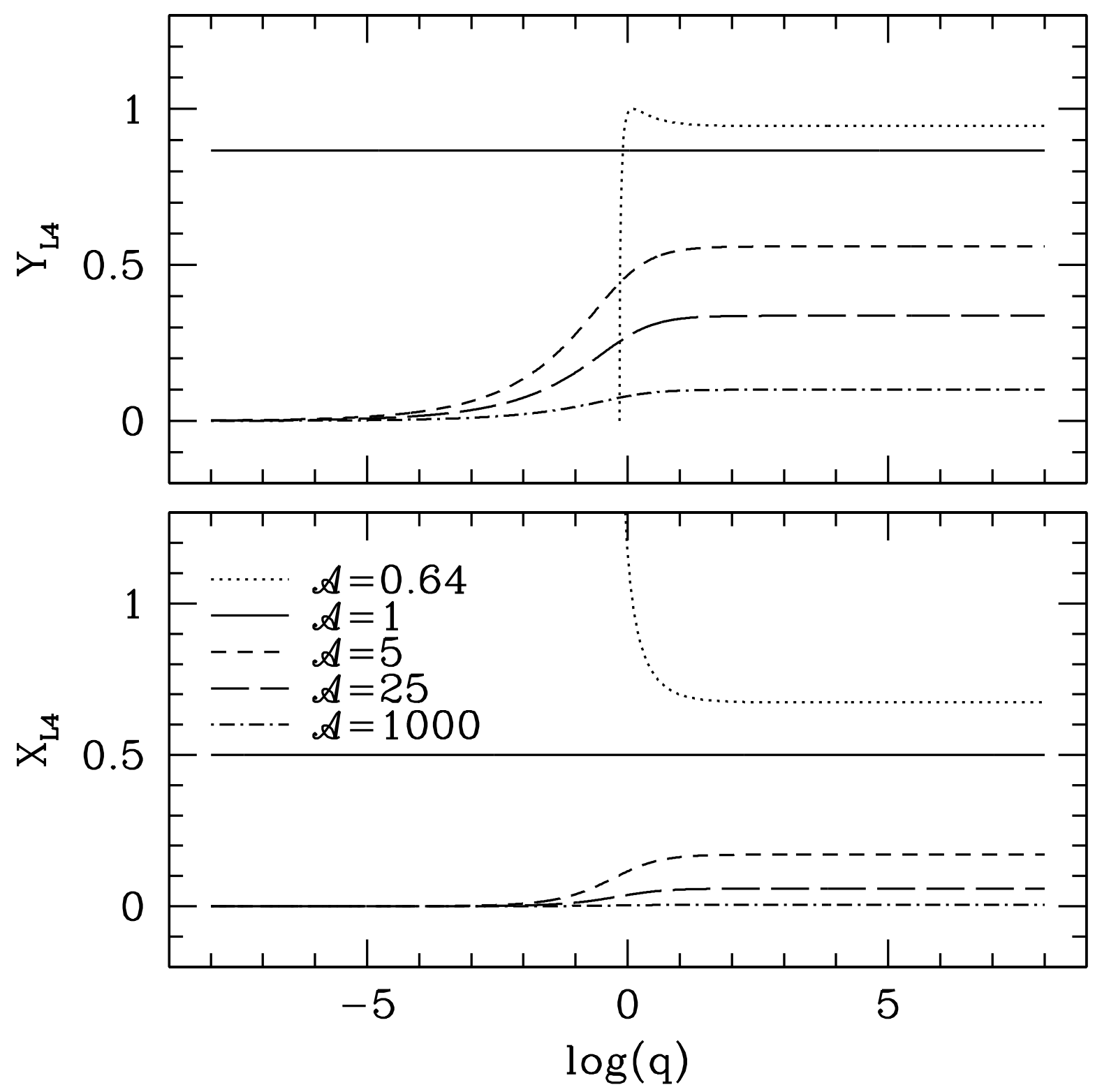

Fig. 5.- The $X_{D}$ and $Y_{D}$ coordinates of the leading triangular Lagrangian point, $L_{4}$, as a function of $q$ for different values of $\mathcal{A}$. The dotted line terminates near $q=0.69$ below which $L_{4}$ does not exist for $\mathcal{A}=0.64$. The $X_{D}$ and $Y_{D}$ coordinates of the trailing Lagrangian point, $L_{5}$, is identical to that of $L_{4}$, except that $Y_{D}$ has the opposite sign for $L_{5}$ as for $L_{4}$. 
The dependence of the $X_{D}$ and $Y_{D}$ coordinates of $L_{4}$ on $q$ and $\mathcal{A}$ is illustrated in the bottom and top panels of Fig. 5, respectively. Since $X_{L 5}=X_{L 4}$ and $Y_{L 5}=-Y_{L 4}$, the dependence of the position of $L_{5}$ on $q$ and $\mathcal{A}$ is similar to that of $L_{4}$. For a given value of $\mathcal{A}>1$, both $X_{L 4}$ and $Y_{L 4}$ increase with increasing values of $q$. The coordinates furthermore asymptote to constant values for both small and large values of $q$. The asymptotic behavior can be understood from Eq. (34) which reduces to

$$
X_{L 4} \approx 0.5\left(\frac{q}{\mathcal{A}-1}\right)^{2 / 3}
$$

for small values of $q$, and

$$
X_{L 4} \approx 0.5 \mathcal{A}^{-2 / 3}
$$

for large values of $q$. Thus, in the limit of small $q, X_{L 4} \rightarrow 0$ when $\mathcal{A}>1$. For $\mathcal{A}<1$, the $L_{4}$ point no longer exists for all values of $q$. E.g., when $\mathcal{A}=0.64$ (the dotted line in Fig. 5 ), the $L_{4}$ point only exists for $q>0.69$ (see Eq. 36). However, when the $L_{4}$ point exists, $X_{L 4}$ still asymptotes to the value given by Eq. (38) for large values of $q$. Finally, for a given value of $q, X_{L 4}$ and $Y_{L 4}$ decrease with increasing values of $\mathcal{A}$. In addition, it is interesting to note that for $\mathcal{A}>1, L_{4}$ and $L_{5}$ are always located inside the binary orbit, while for $\mathcal{A}<1, L_{4}$ and $L_{5}$ are located outside the binary orbit.

\subsection{Stability}



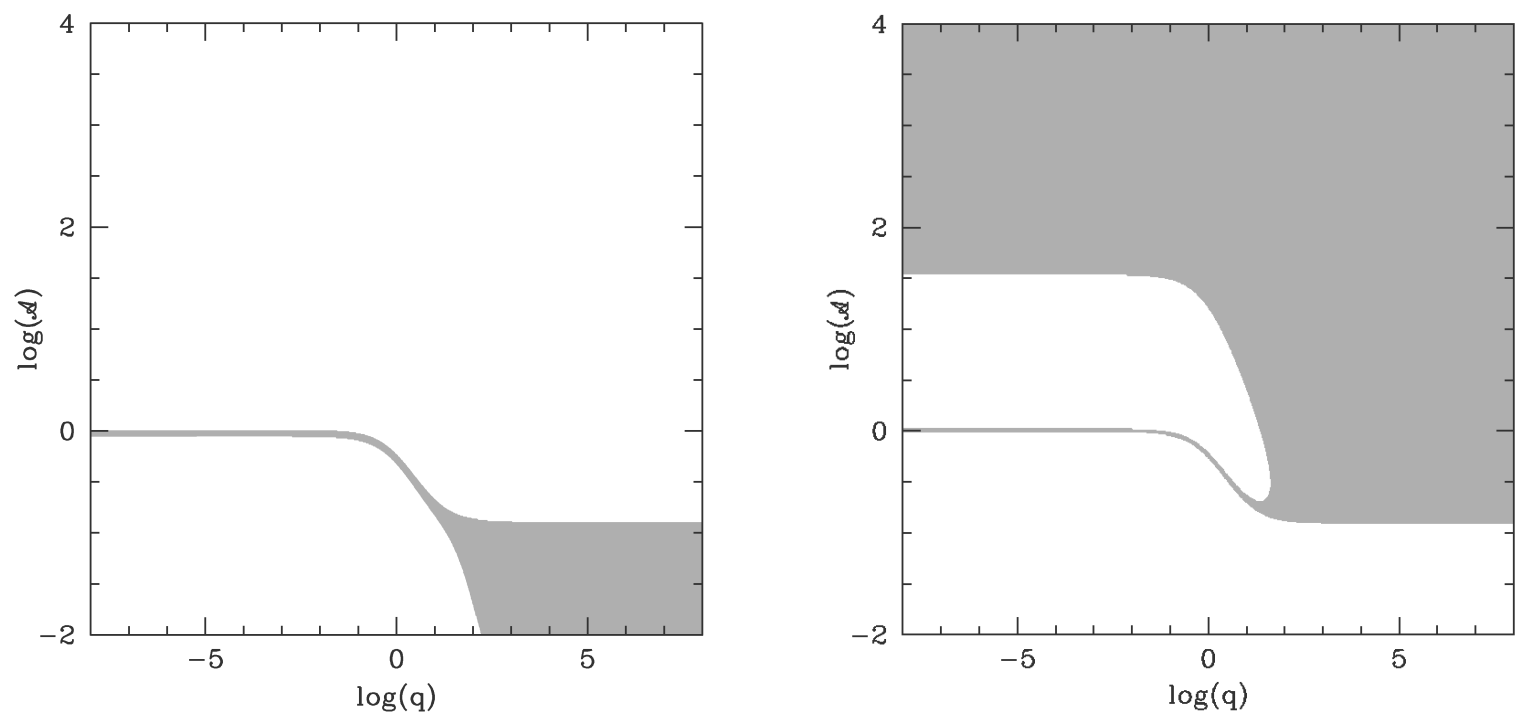

Fig. 6. - Stability regions (gray-shaded) for the Lagrangian points $L_{2}$ (left) and $L_{4}, L_{5}$ (right) in the $(q, \mathcal{A})$-plane. The $L_{2}$ stability region remains always at $\log \mathcal{A}<0$, in agreement with the known results for circular, synchronous binaries $(\log \mathcal{A}=0)$ for which $L_{2}$ is always unstable. The stability regions for $L_{4}$ and $L_{5}$ are identical to each other since the stability conditions given by Eqs. (39)-(41) are dependent only on the square of the $Y_{D}$ coordinate. 
Following the methodology described by Murray \& Dermott (2000) (Chapter 3.7) for circular, synchronous binaries, we examine the stability of the Lagrangian points in eccentric, non-synchronous systems. We employ a linear stability analysis where we perturb and linearize the equation governing the motion of a mass element in the binary potential and follow the behavior of an element given a small displacement from one of the Lagrangian points. If the solution to the perturbed equation of motion is oscillatory, the Lagrange point is stable to small perturbations. All other solutions imply instability. For stability, then, it follows that all three of the following conditions on the second derivatives of the dimensionless potential $V_{D}$ must be satisfied. These are

$$
\begin{aligned}
B^{2} & >4 C \\
\sqrt{B^{2}-4 C} & \leq B \\
\sqrt{B^{2}-4 C} & \geq-B,
\end{aligned}
$$

where

$$
\begin{aligned}
B & \equiv 4 \mathcal{A}(1+q)+V_{X X}+V_{Y Y}, \\
C & \equiv V_{X X} V_{Y Y}-V_{X Y}^{2},
\end{aligned}
$$

$V_{X X} \equiv \partial^{2} V_{D} / \partial X_{D}^{2}, V_{Y Y} \equiv \partial^{2} V_{D} / \partial Y_{D}^{2}$, and $V_{X Y} \equiv \partial^{2} V_{D} / \partial X_{D} \partial Y_{D}$ are the second derivatives of the potential $V_{D}$ with respect to $X_{D}$ and $Y_{D}$, and the square roots in Eqs. (401) and (41) are assumed to be positive. Examining these conditions, we find that $L_{1}$ and $L_{3}$ are always unstable for any combination of binary parameters, while $L_{2}, L_{4}$, and $L_{5}$ can be stable as well as unstable depending on the values of $q$ and $\mathcal{A}$. The regions in the $(q, \mathcal{A})$-plane where $L_{2}, L_{4}$, and $L_{5}$ are stable and are shown by the gray-shaded areas in Fig. 6 .

For $q \lesssim 0.1$, the $L_{2}$ point is found to be stable for a narrow range of $\mathcal{A}$-values very near, but always less than $\mathcal{A}=1$, while for $q \gtrsim 10$, a broad stable region is found at $\mathcal{A} \lesssim 0.1$ (Fig. [6, left panel). In the latter regime, the potential is very similar to that of a non-rotating single star, except for a small contribution due to the gravitational potential of star 2. Even though this contribution is small, it is significant enough to be the sole cause of this stable region. This is further supported by the fact that, in this regime, the potential at $L_{3}$ is identical to that at $L_{2}$ except for this small contribution, and yet $L_{3}$ is never stable throughout the considered parameter space. Thus, the second body, however small, is the sole cause for the stable regions of $L_{2}$. We note that we do not find any stable points for $\log \mathcal{A}=0$, in agreement with the analysis by Murray \& Dermott (2000) for circular, synchronous binary systems.

The stability region for the triangular Lagrangian points, $L_{4}$ and $L_{5}$, covers a wide range of $q$ and $\mathcal{A}$ values which, for $q \lesssim 0.1$, converges into a narrow stability region near $\mathcal{A}=1$ 
(Fig.6, right panel). Solutions at exactly $\mathcal{A}=1$ exist only for $\log q \leq-1.4$ and $\log q \geq 1.4$, in agreement with the analysis of Murray \& Dermott (2000) for circular, synchronized binaries (see their Eq. 3.145). It is apparent, that the $L_{4}$ and $L_{5}$ stability regions are much larger for eccentric, non-synchronous systems than for circular, synchronous systems. The existence of these stable points is important to study the trapping of test particles such as gas attempting to escape the system or even Trojan asteroids in planetary systems (see Ford \& Gaudi 2006, for a discussion on Trojan asteroids around extrasolar planets). It should also be noted that Lagrangian points within the stable regions shown in Fig. 6 need not remain stable throughout the binary orbit due to the dependence of $\mathcal{A}$ on the mean anomaly (see Eq. 21 and Fig. 31).

\section{The Volume-Equivalent Roche-Lobe Radius}

Under the quasi-static approximation, the maximum size of a star in a close binary is determined by the equipotential surface connected to the inner Lagrangian point $L_{1}$. This surface is commonly referred to as the Roche lobe. Once a star fills its Roche lobe, any further expansion of the star or shrinkage of the lobe results in mass loss from the star. For a circular binary with synchronously rotating component stars, the shape and volume of the Roche lobe depend solely on the mass ratio, $q$, of the system. For eccentric binaries with non-synchronous component stars, the shape and volume of the Roche lobe also depend

upon the eccentricity, the true anomaly, and the degree of asynchronism. As before, we group these dependencies into the parameter $\mathcal{A}(f, e, \nu)$ (see Eq. 21). 


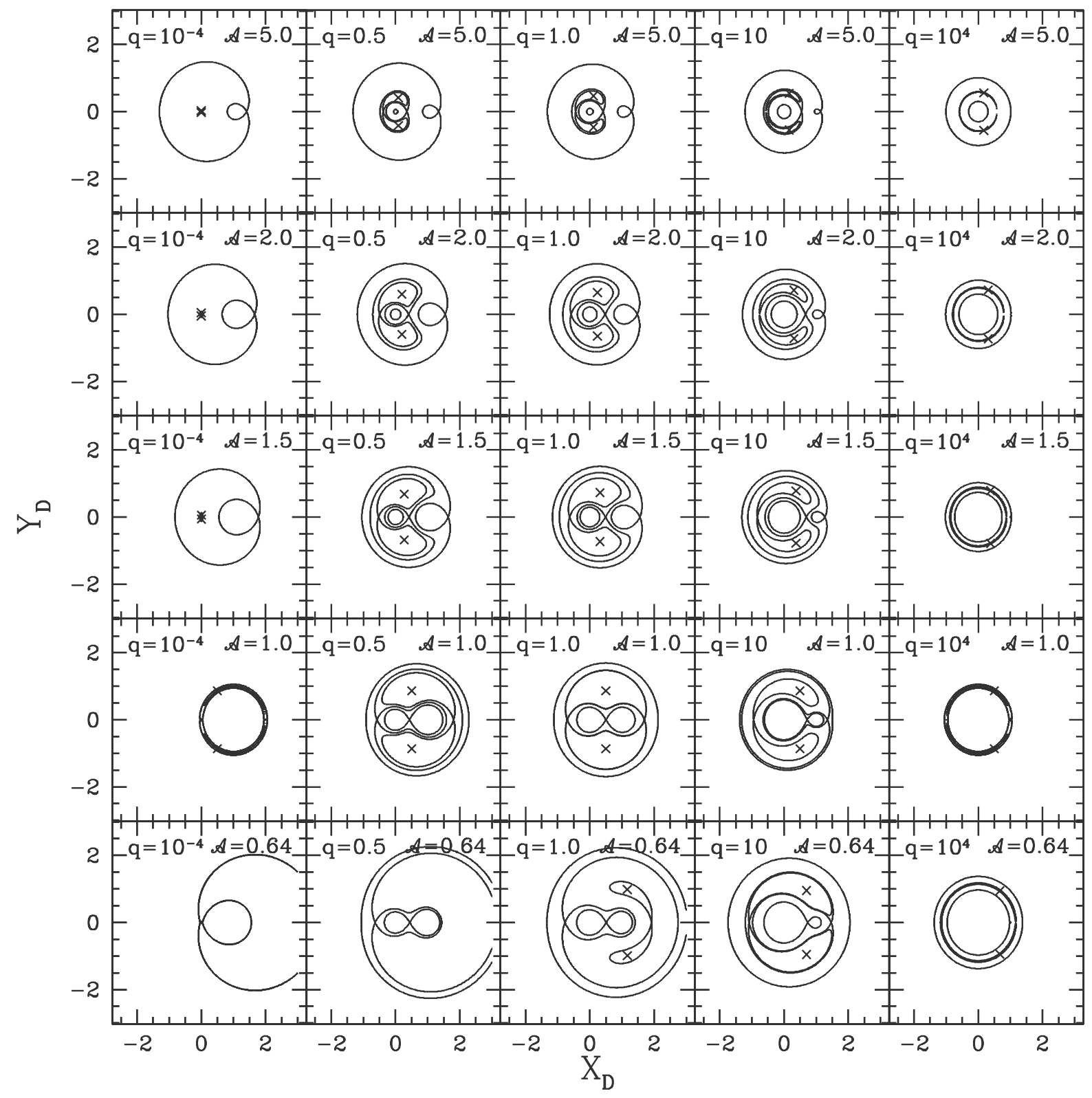

Fig. 7.- Instantaneous equipotential surfaces of $V_{D}$ given by Eq. (20) in the $Z=0$ plane for different mass ratios $q=M_{1} / M_{2}$ and values of the parameter $\mathcal{A}(f, e, \nu)$. The contours shown correspond to the equipotential surfaces passing through the co-linear Lagrangian points $L_{1}$, $L_{2}$, and $L_{3}$. The locations of the triangular Lagrangian points $L_{4}$ and $L_{5}$, if they exist, are marked with crosses. Star 1 is located at $\left(X_{D}, Y_{D}\right)=(0,0)$, and star 2 at $\left(X_{D}, Y_{D}\right)=(1,0)$. The $\mathcal{A}=1.0$ panels correspond to circular binaries with synchronously rotating component stars. For $\mathcal{A}=0.64, L_{4}$ and $L_{5}$ only exist for $q>0.69$ (see Eq. [36). 
The variations in the shape and size of the binary equipotential surfaces are shown in Fig. 7 as functions of $q$ and $\mathcal{A}$. The contours shown correspond to the cross-sections of the equipotential surfaces passing through the co-linear Lagrangian points with the $Z_{D}=0$ plane. The locations of the triangular Lagrangian points, if they exist, are marked with crosses. For large mass ratios, the equipotential surfaces resemble that of a rotating single star regardless of the value of $\mathcal{A}$. For smaller mass ratios, the structure of the equipotential surfaces becomes more complex and the detailed shapes become more sensitive to the value of $\mathcal{A}$. A particularly interesting observation is that for $\mathcal{A}>1$ the equipotential surface passing through $L_{1}$ may "open up" and no longer enclose star 24 . This geometry may facilitate mass loss from the system through $L_{2}$ when star 1 fills its Roche lobe and transfers mass to star 2 through $L_{1}$. We will discuss this in more detail in the next section. For values of $\mathcal{A} \gtrsim 10$, the centrifugal term dominates the potential $V_{D}$, so that the equipotential surfaces resemble those of a rotating single star regardless of the mass ratio $q$.

The equipotential surfaces shown in Fig. 7 differ considerably from those of Regös et al. (2005). In particular, the orbital parameters adopted in Figs. 3 and 4 of that paper yield $\mathcal{A}$-values of 1.4 and 1.8 , respectively, which may be compared to the $\mathcal{A}=1.5$ and $\mathcal{A}=2.0$ panels for $q=0.5$ in Fig. 7. The differences may be attributed to the erroneous use of the Pringle \& Wade (1985) potential in Regös et al. (2005) which is only valid for binaries with circular orbits.

In order to determine whether or not a star fills its critical Roche lobe, a full threedimensional treatment of stellar and binary evolution is, in principle, required. Such a treatment is, however, prohibitively computationally expensive. Roche-lobe overflow is therefore usually studied using one-dimensional stellar and binary evolution codes in which the Roche lobe of a star is approximated by a sphere of equivalent volume. The star of radius $\mathcal{R}_{1}$ is then assumed to overflow its Roche lobe and transfer mass to its companion when $\mathcal{R}_{1}>\mathcal{R}_{L}$, where $\mathcal{R}_{L}$ is the radius of the sphere with volume equal to that of the Roche lobe.

\footnotetext{
${ }^{4}$ Note that this "opening up" of the Roche potential has also been seen in studies of radiation effects on the shape of the equipotential surfaces. See Schuerman (1972) and Vanbeveren (1977).
} 


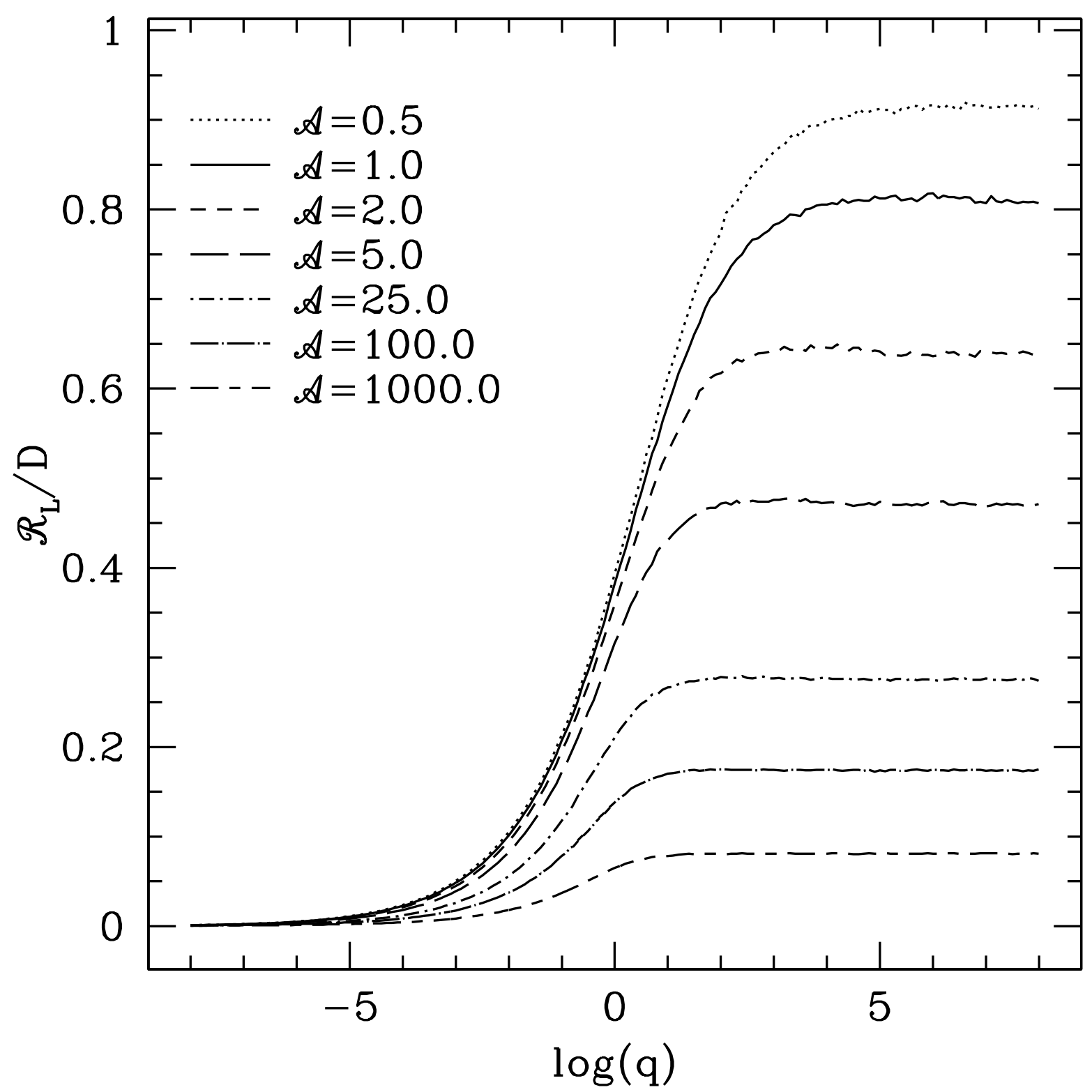

Fig. 8. - The volume-equivalent Roche lobe radius, $\mathcal{R}_{L}$ in units of the instantaneous distance $D(t)$ between the binary components as a function of $\log q$, for a range of $\mathcal{A}$-values. Small fluctuations in the curves are due to the finite errors in the Monte Carlo integration method used to calculate the volume of the Roche lobe. 
In Fig. 8, we show the volume-equivalent Roche lobe radius in units of the instantaneous distance between the binary components calculated via Monte Carlo volume integration as a function of the mass ratio, $q$, for a range of $\mathcal{A}$-values. We note that our calculation of the Roche lobe radius agrees with that of Vanbeveren (1977) to within a few percent in the regime in which our parameter spaces intersect (circular, non-synchronous orbits where the effects of radiation pressure have been neglected). Comparison of Fig. 8 with Fig. 4 shows that $\mathcal{R}_{L}$ as a function of $q$ is similar in shape to $L_{1}$ as a function of $q$, which is expected since it is the potential at $L_{1}$ which defines the size and shape of the Roche lobe. For small values of $q, \mathcal{R}_{L}$ asymptotes to zero independent of $\mathcal{A}$ because the location of $L_{1}$ asymptotes to the position of star 1 . For large values of $q, \mathcal{R}_{L}$ asymptotes to an $\mathcal{A}$-dependent value.

A simple and accurate fitting formula for the volume-equivalent Roche Lobe radius of a star in a circular binary with synchronously rotating component stars has been provided by Eggleton (1983):

$$
\mathcal{R}_{L, \text { circ }}^{\text {Egg }}=a \frac{0.49 q^{2 / 3}}{0.6 q^{2 / 3}+\ln \left(1+q^{1 / 3}\right)},
$$

where $a$ is the radius of the circular orbit. For lack of a better treatment, this formula is often extrapolated to binaries with eccentric orbits. In particular, Roche lobe radii of stars at the periastron of a binary orbit are commonly approximated by

$$
\mathcal{R}_{L, \text { peri }}^{\text {Egg }}=a(1-e) \frac{0.49 q^{2 / 3}}{0.6 q^{2 / 3}+\ln \left(1+q^{1 / 3}\right)},
$$

where $a(1-e)$ is the periastron distance of the binary orbit. We can further extend this generalization and apply it to arbitrary orbital phases by replacing the periastron distance between the stars with the current orbital separation, $D(t)$. Thus,

$$
\mathcal{R}_{L}^{\mathrm{Egg}}=D(t) \frac{0.49 q^{2 / 3}}{0.6 q^{2 / 3}+\ln \left(1+q^{1 / 3}\right)} .
$$




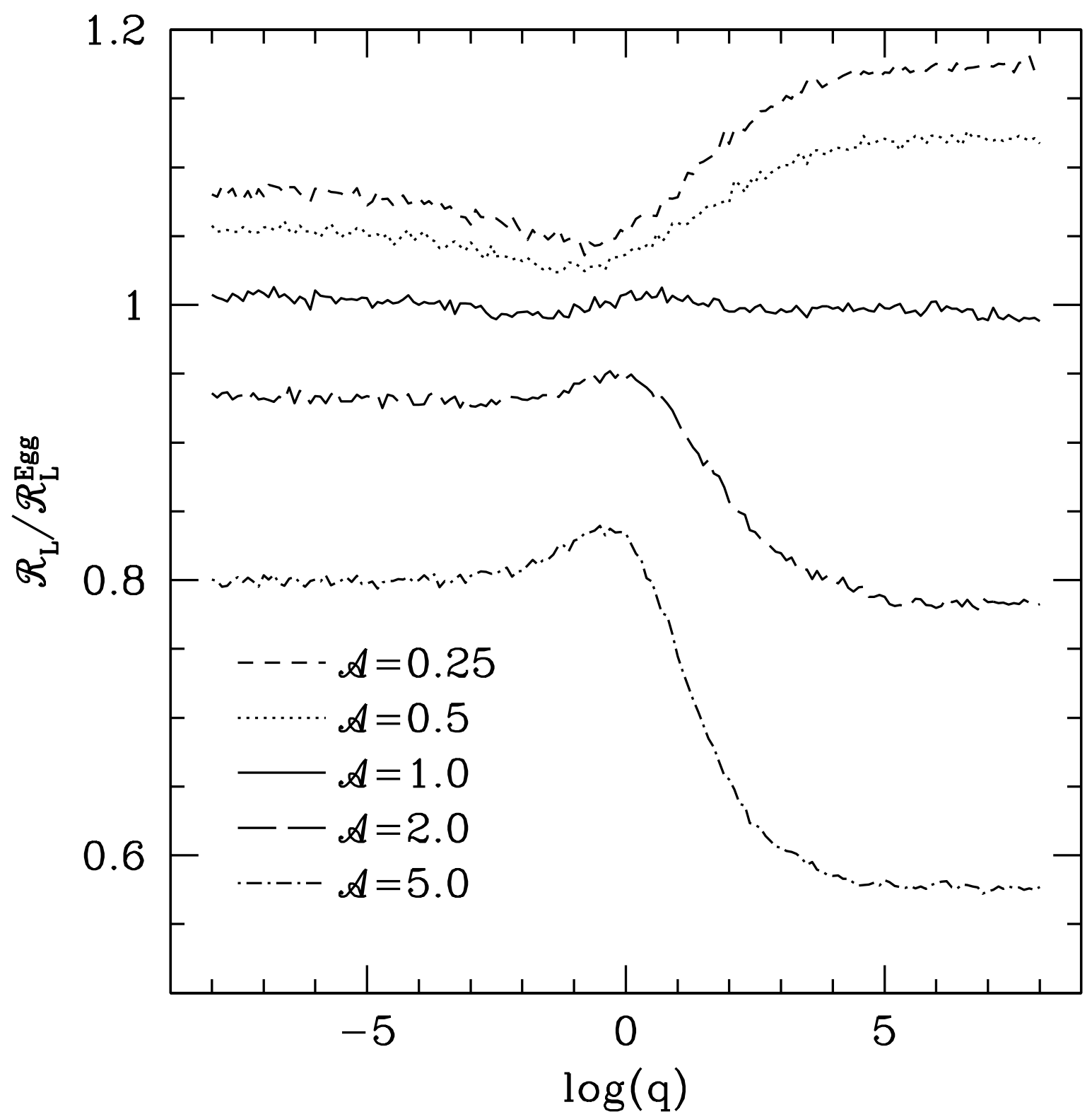

Fig. 9.- The ratio of the volume-equivalent Roche Lobe radius at periastron obtained by numerically calculating the volume of the Roche lobe to the volume-equivalent Roche Lobe radius obtained from Eq. (46). Small fluctuations in the curves are due to the finite errors in the Monte Carlo integration method used to calculate the volume of the Roche lobe. 
In Fig. 9, we show the ratio of the volume-equivalent Roche Lobe radius at periastron calculated through a Monte Carlo integration method to the volume-equivalent Roche Lobe radius determined by means of Eq. (46). For $\mathcal{A} \approx 1$, we find Eq. (46) to be accurate to within a few percent over a wide range of $q$-values, confirming previous statements on the accuracy of the equation based on smoothed particle hydrodynamics calculations (Faber et al. 2005; Regös et al. 2005). For a given value of $\mathcal{A}$, the ratio $\mathcal{R}_{L} / \mathcal{R}_{L}^{\text {Egg }}$ is closest to unity near $q=1$ and indicates better agreement between $\mathcal{R}_{L}$ and $\mathcal{R}_{L}^{\text {Egg }}$ for $q<1$ than for $q>1$, especially for large values of $\mathcal{A}$ (by almost a factor of 2 as shown in Fig. 9). For a given value of $q$, the agreement between $\mathcal{R}_{L}$ and $\mathcal{R}_{L}^{\text {Egg }}$ furthermore becomes progressively worse as $\mathcal{A}$ differs from unity.

Hence, a star rotating synchronously with the orbital angular velocity at periastron in an eccentric binary (so that $1 \lesssim \mathcal{A} \lesssim 2$ for $e<0.9$ ) has a smaller volume-equivalent Roche Lobe radius at periastron than a star rotating synchronously in a circular orbit with radius equal to the periastron distance of the eccentric binary. As shown in Fig. 9, this decrease in $\mathcal{R}_{L}$ may be substantially smaller than the $(1-e)$ factor introduced in Eq. (45). Consequently, the maximum size of star 1 is smaller in an eccentric binary and mass transfer from star 1 to star 2 may begin earlier than in a circular binary with the same instantaneous distance between the component stars.

For ease of use, we provide a fitting formula for $\mathcal{R}_{L} / \mathcal{R}_{L}^{\text {Egg }}$ as a function of $q$ and $\mathcal{A}$. In order to keep the accuracy of the fit better than $1 \%$, it is necessary to divide the parameter space into six different regimes. The formulae below are accurate to better than $1 \%$ for $-8 \leq \log q \leq 8$ and $-2 \leq \log \mathcal{A} \leq 4$, and, in most cases, the accuracy is better than $0.5 \%$.

$$
\begin{gathered}
\frac{\mathcal{R}_{L}}{\mathcal{R}_{L}^{\mathrm{Egg}}} \quad[\log q \geq 0 ; \log \mathcal{A} \leq-0.1]= \\
1.226-0.21 \mathcal{A}-0.15(1-\mathcal{A}) \exp \left[(0.25 \mathcal{A}-0.3)(\log q)^{1.55}\right] \\
\frac{\mathcal{R}_{L}}{\mathcal{R}_{L}^{\mathrm{Egg}}} \quad[\log q \leq 0 ; \log \mathcal{A} \leq-0.1]= \\
\quad 1+0.11(1-\mathcal{A})-0.05(1-\mathcal{A}) \exp \left[-(0.5(1+\mathcal{A})+\log q)^{2}\right] \\
\frac{\mathcal{R}_{L}}{\mathcal{R}_{L}^{\mathrm{Egg}}} \quad[\log q \leq 0 ;-0.1 \geq \log \mathcal{A} \leq 0.2]=g_{0}(\mathcal{A})+g_{1}(\mathcal{A}) \log q+g_{2}(\mathcal{A})(\log q)^{2}
\end{gathered}
$$




$$
\begin{aligned}
& g_{0}(\mathcal{A})=0.9978-0.1229 \log \mathcal{A}-0.1273(\log \mathcal{A})^{2} \\
& g_{1}(\mathcal{A})=0.001+0.02556 \log \mathcal{A} \\
& g_{2}(\mathcal{A})=0.0004+0.0021 \log \mathcal{A}
\end{aligned}
$$

$$
\begin{aligned}
\frac{\mathcal{R}_{L}}{\mathcal{R}_{L}^{\text {Egg }}} \quad[\log q \geq 0 ;-0.1 \geq \log \mathcal{A} \leq 0.2]=h_{0}(\mathcal{A})+h_{1}(\mathcal{A}) \log q+h_{2}(\mathcal{A})(\log q)^{2} \\
h_{0}(\mathcal{A})=1.0071-0.0907 \log \mathcal{A}-0.0495(\log \mathcal{A})^{2} \\
h_{1}(\mathcal{A})=-0.004-0.163 \log \mathcal{A}-0.214(\log \mathcal{A})^{2} \\
h_{2}(\mathcal{A})=0.00022-0.0108 \log \mathcal{A}-0.02718(\log \mathcal{A})^{2}
\end{aligned}
$$

$$
\begin{aligned}
\frac{\mathcal{R}_{L}}{\mathcal{R}_{L}^{\text {Egg }}} \quad[\log q & \leq 0 ; \log \mathcal{A} \geq 0.2]=i_{0}(\mathcal{A})+i_{1}(\mathcal{A}) \exp \left[-i_{2}(\mathcal{A})\left(\log q+i_{3}(\mathcal{A})\right)^{2}\right] \\
i_{0}(\mathcal{A}) & =\frac{6.3014(\log \mathcal{A})^{1.3643}}{\exp \left[2.3644(\log \mathcal{A})^{0.70748}\right]-1.4413 \exp \left[-0.0000184(\log \mathcal{A})^{-4.5693}\right]} \\
i_{1}(\mathcal{A}) & =\frac{\log \mathcal{A}}{0.0015 \exp \left[8.84(\log A)^{0.282}\right]+15.78} \\
i_{2}(\mathcal{A}) & =\frac{1+0.036 \exp \left[8.01(\log \mathcal{A})^{0.879}\right]}{0.105 \exp \left[7.91(\log \mathcal{A})^{0.879}\right]} \\
i_{3}(\mathcal{A}) & =\frac{0.991}{1.38 \exp \left[-0.035(\log \mathcal{A})^{0.76}\right]+23.0 \exp \left[-2.89(\log \mathcal{A})^{0.76}\right]}
\end{aligned}
$$

$$
\begin{aligned}
\frac{\mathcal{R}_{L}}{\mathcal{R}_{L}^{\mathrm{Egg}}} \quad[\log q \geq 0 ; \log \mathcal{A} \geq 0.2]=j_{0}(\mathcal{A})+j_{1}(\mathcal{A}) \exp \left[-j_{2}(\mathcal{A})(\log q)^{j_{3}(\mathcal{A})}\right] \\
j_{0}=\frac{1.895(\log \mathcal{A})^{0.837}}{\exp \left[1.636(\log A)^{0.789}\right]-1} \\
j_{1}=\frac{4.3(\log \mathcal{A})^{0.98}}{\exp \left[2.5(\log \mathcal{A})^{0.66}\right]+4.7} \\
j_{2}=\frac{1}{8.8 \exp \left[-2.95(\log \mathcal{A})^{0.76}\right]+1.64 \exp \left[-0.03(\log \mathcal{A})^{0.76}\right]} \\
j_{3}=0.256 \exp \left[-1.33(\log \mathcal{A})^{2.9}\right]\left(5.5 \exp \left[1.33(\log \mathcal{A})^{2.9}\right]+1\right)
\end{aligned}
$$




\section{Systemic mass loss}

As mentioned in the previous section, once star 1 fills its Roche lobe, any further stellar expansion or orbital contraction may cause the star to begin losing mass through $L_{1}$. In the standard case of a circular synchronous binary, any matter flowing from star 1 through $L_{1}$ will, in the absence of other non-gravitational or rotational effects, be captured by star 2 , either forming a disk of material or falling directly onto its surface. In the case of an eccentric and/or non-synchronous binary, the geometry of the equipotential surfaces is such that this is not necessarily the case. 


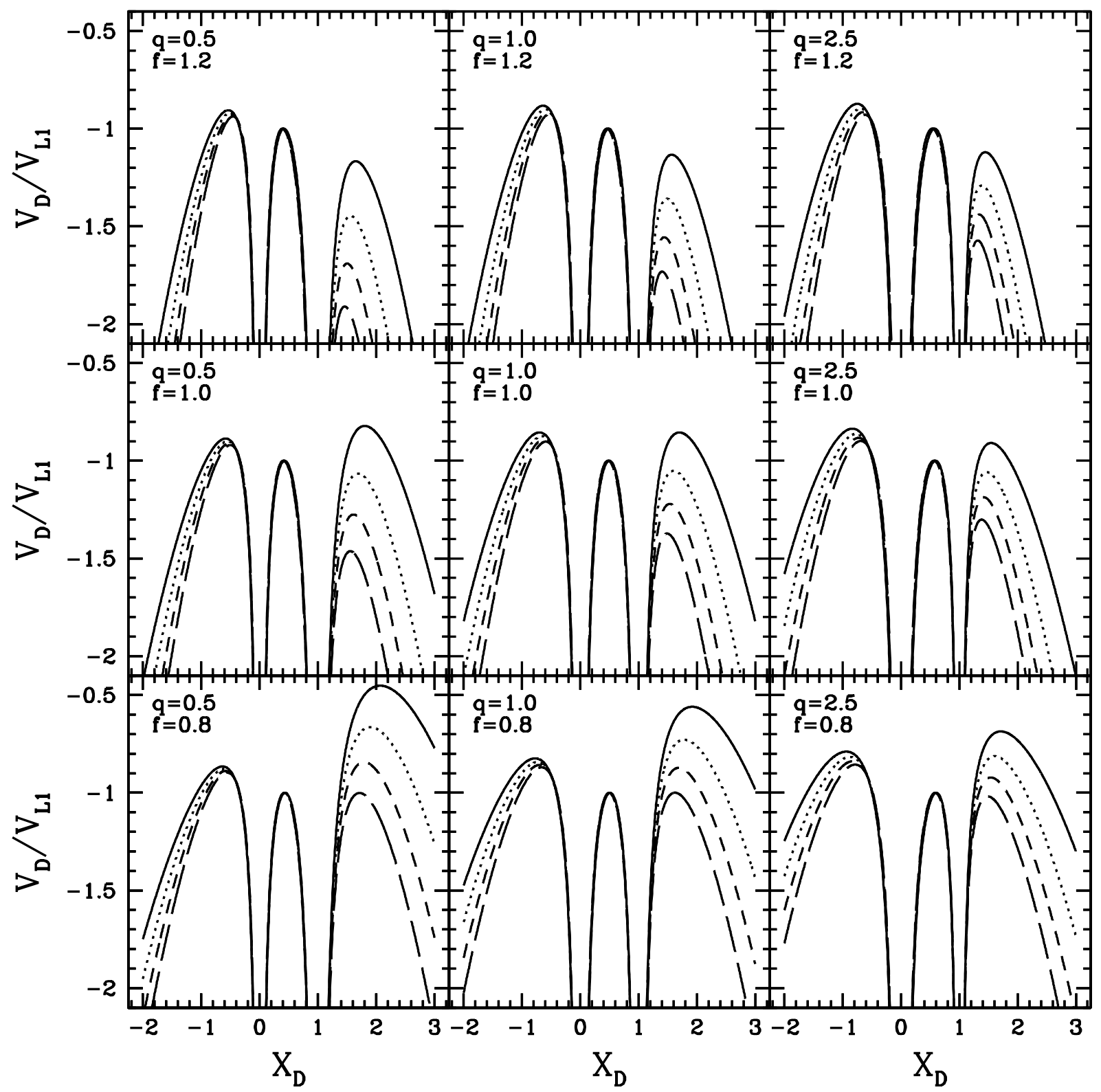

Fig. 10.- Height of the dimensionless potential $V_{D}$ at periastron along the $X_{D}$-axis $\left(Y_{D}=\right.$ $\left.Z_{D}=0\right)$ for different values of the mass ratio, orbital eccentricity, and ratio of rotational to orbital angular velocity at periastron. Different line types in each panel correspond to the orbital eccentricities $e=0$ (solid lines), $e=0.3$ (dotted lines), $e=0.6$ (short-dashed lines), and $e=0.9$ (long-dashed lines). In each panel, the maxima in the curves from left to right correspond to the $L_{3}, L_{1}$, and $L_{2}$ point. Star 1 is located at $X_{D}=0$ and star 2 at $X_{D}=1$. For ease of comparison, the curves have been normalized to the height of the potential at $L_{1}$. 
In particular, we have seen from Fig. 7 that for significantly large $\mathcal{A}$, the equipotential surface passing through $L_{1}$ no longer encloses star 2. Matter transferred from star 1 to star 2 through the inner Lagrangian point $L_{1}$ may therefore be lost from the system. Whether or not this happens depends on the particle trajectories in the mass-transfer stream which we will address in detail in a forthcoming paper. For the purpose of this paper, we restrict ourselves to comparing the height of the potential at the three co-linear Lagrangian points as an indicator of possible mass loss scenarios in eccentric binaries.

Since the first occurrence of mass transfer in eccentric binaries is expected to take place when the stars are closest to each other, we focus our discussion on the height of the potential at the periastron of the binary orbit. In addition, the graphical representation of the height of the potential turns out to be clearer in terms of the mass ratio, orbital eccentricity, and ratio of rotational to orbital angular velocity at periastron than in terms of the parameter $\mathcal{A}$. Hence, the height of the potential $V_{D}$ at periastron along the line connecting the mass centers of the component stars (the $X_{D}$-axis) is shown in Fig. 10 as a function of $q$, $e$, and $f$. To facilitate the comparison of the relative height of the potential at the co-linear Lagrangian points, the potential is furthermore normalized to its value at the inner Lagrangian point $L_{1}$.

For the considered ranges of mass ratios, eccentricities, and rotation rates, the relative height of the potential at $L_{2}$ depends most sensitively on $e$ and $f$, while the relative height of the potential at $L_{3}$ is largely constant over the considered range of parameters. For a constant $f$ and $q$, an increasing eccentricity tends to decrease the relative height of the potential. The decrease is stronger for larger $f$-values and smaller $q$-values.

For the considered parameter ranges, the shape of the normalized potential near $L_{1}$ is furthermore highly insensitive to the orbital parameters, while the height of the potential, as well as the position of the peak, can change for both $L_{2}$ and $L_{3}$. Most interestingly, the potential at $L_{2}$ may be higher than, equal to, or lower than the potential at the $L_{1}$ point. Thus, matter flowing from star 1 through the $L_{1}$ point will not necessarily be captured by star 2, and may be energetic enough to escape the system. As such, it is possible that mass transfer through $L_{1}$ can be significantly non-conservative due to the geometry of the equipotential surfaces in eccentric binaries, but a calculation of the amount of matter lost from the system will require detailed particle trajectories, which is beyond the scope of this paper. We also note that the value of the potential at $L_{3}$ can, for certain values of the orbital parameters, be very near to the value of the potential at $L_{1}$. In cases such as this, it may be possible for a small amount of matter also to be lost from star 1 through $L_{3}$ in addition to that lost through $L_{1}$. However, contrary to Regös et al. (2005), we find the potential at $L_{3}$ to be always higher than the potential at $L_{1}$, so that mass loss through $L_{3}$ never takes place 
prior to mass loss through $L_{1} 5$. This difference may be attributed to the erroneous use of the Pringle \& Wade (1985) potential by Regös et al. (2005).

\section{Summary}

In this paper, we present a detailed investigation of the existence and properties of equipotential surfaces and Lagrangian points in eccentric binary star and planetary systems with non-synchronously rotating component stars or planets. The analysis is valid as long as the time scale of any tidally induced oscillations is long compared to the dynamical time scale of the component stars or planets. Once the foundation of the potential is laid, we (i) solve for the positions of the three co-linear and two triangular Lagrangian points, (ii) study the stability of the points, (iii) provide a semi-analytical fit for the general solution of the volume-equivalent Roche lobe radius that allows the determination of when mass transfer is initiated, and (iv) discuss the role of the potential geometry and structure in inducing the possibility of systemic mass loss. Throughout the analysis we compare our results to the well-studied case of synchronous binaries with circular orbits and quantify the differences, which turn out to be significant depending on the binary properties.

Our study has been motivated by the need for characterizing the gravitational potential for the general case of eccentric, non-synchronous binaries when considering interactions (mass transfer or mass and angular momentum loss) that occur in binaries before full synchronization and circularization has been achieved. The analysis presented here can be applied to both stellar and planetary systems for a wide range of problems. We plan to use the results to study the ballistic motion of test particles in the potential, assess the conditions under which mass transfer may be conservative or not, and eventually apply the results in the development of a theoretical framework that allows modeling of the orbital evolution of interacting, eccentric, non-synchronous binaries.

We thank Chris Belczynski and Fred Rasio for useful discussions, as well as an anonymous referee for the encouragement to complete our analytic fits for the Roche lobe radius.. This work is supported by a NASA GSRP Fellowship to JS, a Packard Foundation Fellowship in Science and Engineering and a NSF CAREER grant (AST-0449558) to VK.

\footnotetext{
${ }^{5}$ Note that Regös et al. (2005) adopt a nomenclature for $L_{2}$ and $L_{3}$ that is opposite to ours.
} 


\section{REFERENCES}

Avni, Y. 1976, ApJ, 209, 574

Avni, Y., \& Schiller, N. 1982, ApJ, 257, 703

Bayne, G., Tobin, W., Pritchard, J. D., Bond, I., Pollard, K. R., Besier, S. C., Noda, S., Sumi, T., Yanagisawa, T., Sekiguchi, M., Honda, M., Muraki, Y., Takeuti, M., Hearnshaw, J. B., Kilmartin, P. M., Dodd, R. J., Sullivan, D. J., \& Yock, P. C. M. 2002, MNRAS, 331, 609

Bayne, G. P., Tobin, W., Pritchard, J. D., Pollard, K. R., \& Albrow, M. D. 2004, MNRAS, 349,833

Bildsten, L., Chakrabarty, D., Chiu, J., Finger, M. H., Koh, D. T., Nelson, R. W., Prince, T. A., Rubin, B. C., Scott, D. M., Stollberg, M., Vaughan, B. A., Wilson, C. A., \& Wilson, R. B. 1997, ApJS, 113, 367

Clausen, J. V., Storm, J., Larsen, S. S., \& Giménez, A. 2003, A\&A, 402, 509

Domiciano de Souza, A., Kervella, P., Jankov, S., Abe, L., Vakili, F., di Folco, E., \& Paresce, F. 2003, A\&A, 407, L47

Eggleton, P. P. 1983, ApJ, 268, 368

Faber, J. A., Rasio, F. A., \& Willems, B. 2005, Icarus, 175, 248

Ford, E. B., \& Gaudi, B. S. 2006, ArXiv Astrophysics e-prints

Habets, G. M. H. J., \& Zwaan, C. 1989, A\&A, 211, 56

Hegedüs, T., Giménez, A., \& Claret, A. 2005, in ASP Conf. Ser. 333: Tidal Evolution and Oscillations in Binary Stars, ed. A. Claret, A. Giménez, \& J.-P. Zahn, 88-+

Hilditch, R. W., Howarth, I. D., \& Harries, T. J. 2005, MNRAS, 357, 304

Jones, H. R. A., Butler, R. P., Tinney, C. G., Marcy, G. W., Carter, B. D., Penny, A. J., McCarthy, C., \& Bailey, J. 2006, MNRAS, 369, 249

Kruszewski, A. 1963, Acta Astronomica, 13, 106

Levato, H. 1974, A\&A, 35, 259

Limber, D. N. 1963, ApJ, 138, 1112 
Lubow, S. H. 1979, ApJ, 229, 1008

Marcy, G. W., \& Butler, R. P. 1995, IAU Circ. No. 6251

Matese, J. J., \& Whitmire, D. P. 1983, ApJ, 6, 776

Mathieu, R. D., Meibom, S., \& Dolan, C. J. 2004, ApJ, 602, L121

Mayor, M., \& Queloz, D. 1995, Nature, 378, 355

Meibom, S., \& Mathieu, R. D. 2005, ApJ, 620, 970

Meibom, S., Mathieu, R. D., \& Stassun, K. G. 2006, ArXiv Astrophysics e-prints

Murray, C. D., \& Dermott, S. F. 2000, Solar System Dynamics (Solar System Dynamics, by C.D. Murray and S.F. Dermott. ISBN 0521575974. http://www.cambridge.org/us/catalogue/catalogue.asp?isbn=0521575974. Cambridge, UK: Cambridge University Press, 2000.)

Peters, P. C. 1964, Physical Review, 136, 1224

Petrova, A. V., \& Orlov, V. V. 1999, AJ, 117, 587

Plavec, M. 1958, Mémoires de la Société Royale des Sciences Liège, 20, 411

Polfliet, R., \& Smeyers, P. 1990, A\&A, 237, 110

Popper, D. M., \& Plavec, M. 1976, ApJ, 205, 462

Pourbaix, D., Tokovinin, A. A., Batten, A. H., Fekel, F. C., Hartkopf, W. I., Levato, H., Morrell, N. I., Torres, G., \& Udry, S. 2004, A\&A, 424, 727

Pringle, J. E., \& Wade, R. A. 1985, Interacting binary stars (Interacting Binary Stars)

Regös, E., Bailey, V. C., \& Mardling, R. 2005, MNRAS, 358, 544

Savonije, G. J. 1978, A\&A, 62, 317

Schuerman, D. W. 1972, Ap\&SS, 19, 351

Sepinsky, J., Willems, B., \& Kalogera, V. 2007, in press

Shapley, H. 1913, ApJ, 38, 158

Spruit, H. C. 1999, A\&A, 341, L1 
Spruit, H. C., \& Phinney, E. S. 1998, Nature, 393, 139

Struve, O. 1950, Stellar evolution, an exploration from the observatory. (Princeton, Princeton University Press, 1950.)

Tout, C. A., Pols, O. R., Eggleton, P. P., \& Han, Z. 1996, MNRAS, 281, 257

van Hamme, W., \& Wilson, R. E. 1990, AJ, 100, 1981

Vanbeveren, D. 1977, A\&A, 54, 877

Wilson, R. E. 1979, ApJ, 234, 1054

Zahn, J. P. 1970, A\&A, 4, 452

Zahn, J.-P. 1975, A\&A, 41, 329 